\title{
Spinning polariton vortices with magnetic field
}

\author{
A. V. Yulin ${ }^{1}$, A. V. Nalitov ${ }^{2,1}$, and I. A. Shelykh ${ }^{2,1}$ \\ ${ }^{1}$ ITMO University, Kronverkskiy prospekt 49, Saint Petersburg 197101, Russia and \\ ${ }^{2}$ Science Institute, University of Iceland, Dunhagi 3, IS-10\%, Reykjavik, Iceland
}

\begin{abstract}
We study the formation dynamics of spinor polariton condensates trapped in ring shaped confining potentials creted by excitonic reservoirs. We consider in detail the interplay of the effective spin-orbit interaction provided by TE-TM splitting of the photonic mode and exciton Zeeman splitting provided by an external magnetic field. We demonstrate that tuning of the trap size obtained by shaping of the external nonresonant and depolarized pumping allows formation of pairs of halfvortices of topological charges $\pm 1 / 2$ in both spin components. Further we show that the probabilities of the realizations of four possible vortex configurations strongly depend on the value of the magnetic field. For certain values of the field the probability of the formation of a vortex with desired topological charge reaches $90 \%$, which opens the possibility of on-demand control of angular momentum of quantum fluids of light with a magnetic field.
\end{abstract}

\section{INTRODUCTION}

Application of a magnetic field dramatically changes the behavior of physical systems. In classical physics, charged particles placed in a magnetic field move along circular orbitals. In the quantum case, the presence of the vector potential affects the phase of a wavefunction of a charged particle leading to such phenomena as splitting of the energy levels corresponding to the different projections of the angular momentum and famous AharonovBohm effect $[1,2]$. In both cases initial symmetry of the clockwise and anti-clockwise rotation is broken, and charged particles are rotated by a magnetic field.

Naturally, for neutral particles magnetic field cannot directly change the orbital motion. However, it can still affect their quantum states by interacting with their spin. If the latter is coupled to the orbital degrees of freedom via spin-orbit interaction, the application of a magnetic field can lead to the appearance of a Berry phase and $\mathrm{U}(1)$ synthetic gauge field, which affects the phase of the wavefunction of a neutral particle in a way similarly to those real magnetic field affects the phase of the wavefunction of a charged particle [3].

In particular, effects of the Berry phase were predicted to play substantial role in excitonic [4] and polaritonic [5-7] systems. The latter lies at the interface between condensed matter physics and optics and will be in focus of the present work.

Exciton-polaritons are hybrid light-matter quasiparticles emerging in the regime of the strong coupling between a photonic mode of a planar semiconductor microcavity and an excitonic resonance in a quantum well embedded in the antinode of a cavity mode [8]. From their photonic component polaritons inherit extremely small effective mass (about $10^{-5}$ of the mass of free electrons) and large coherence length (in the $\mathrm{mm}$ scale) [9]. On the other hand, the presence of an excitonic component results in efficient polariton-polariton interactions, and leads to the extremely strong nonlinear optical response. This makes quantum microcavities a unique laboratory for the study of quantum collective phenomena, such as polariton BEC and superfluidity, at surprisingly high temperatures [10].

An important property of cavity polaritons is their spin (or pseudo-spin) [11], inherited from the spins of QW excitons and cavity photons. Similar to photons, polaritons have two possible spin projections on the structure growth axis corresponding to the two opposite circular polarizations which can be mixed by effective magnetic fields of various origin. Real magnetic field applied along the structure growth axis and acting on the excitonic component splits in energy the polariton states with opposite circular polatizations, while TE-TM splitting of the photonic modes of a planar resonator couples these states to each other via $k$-dependent term, thus playing a role of an effective spin-orbit interaction [11]. The interplay between Zeeman and TE-TM splittings leads to a plethora of intriguing phenomena, in particular in artificial polariton lattices [12-19].

Importantly, polariton-polariton interactions are also spin dependent. Indeed, they stem from the interactions of their excitonic components, which is dominated by the exchange term [20]. This leads to the fact that polaritons of the same circular polarization interact orders of magnitude stronger then polaritons with opposite circular polarizations [21]. Polarization dependent interactions render the system strongly nonlinear, which enables formation of self-sustained nonlinear topological defects such as solitons [22-26] and vortices [27-31]. Their robustness is topologically protected, which makes them readily observable in experiments even in the presence of pump and decay $[32,33]$, and attractive from the point of view of the potential applications [34-36].

In the current work we study a remarkable effect of spinning of polariton vortices formed in nonequilibrium localized polariton condensates. We show that this effect occurs due to the delicate interplay between drivendissipative nature of the system, polarization dependent nonlinearity and breaking of the time reversal symmetry provided by cumulative effect of Zeeman and TE-TM splittings.

The paper is organized as follows. The model for confined nonequilibrium polariton condensates formulated in 
terms of coupled generalized Gross-Pitaevskii equations and rate equations for the incoherent excitonic reservoir density is presented in Section II. Sections II A and II B report on formation of the four stable stable states in numerical simulations and their corresponding probabilities in the absence and in the presence of an external magnetic fields respectively. These observations are discussed in detail in Section III and are qualitatively compared to the results of the semi-phenomenological toy model, introduced in Section III A. Section IV contains the conclusions.

\section{THE MODEL}

Polariton condensate is a driven-dissipative multistable system for which the probabilities of the formation of different macroscopically ordered states from uncondensed phase depend on their occupation growth rates. Typically, the fastest growing mode wins the mode competition and suppresses the others. This can be used in particular to monitor the absolute value of the angular momentum of spontaneously forming condensates [37].

In this paper we investigate the prospect of control over rotation of an emerging condensate by application of an external magnetic field. In contrast to the previous work [37], we demonstrate additional possibility for the selection of preferential rotation direction provided by spinor nature of the polaritons.

We consider the case of a spinor condensate simultaneously trapped and excited by a radially symmetric annular nonresonant pump. The dynamics of the polariton condensate and the exciton reservoir is described by the standard set of coupled driven-dissipative equations of Gross- Pitaevskii type for the order parameter functions $\varphi_{\uparrow(\downarrow)}$ describing the spin-up and spin-down polaritons (so the basis of circular polarizations is used) and the rate equations describing the density of incoherent excitons $\rho_{\uparrow(\downarrow)}$ that create both the effective gain and the repelling potential for the coherent polaritons. In physical units these equation read:

$$
\begin{gathered}
i \hbar \frac{\partial \varphi_{\uparrow(\downarrow)}}{\partial t}=\left[-\frac{\hbar^{2}}{2 m_{e f f}} \nabla^{2}+\frac{i \hbar}{2}\left(R \rho_{\uparrow(\downarrow)}-\gamma_{c}\right)+\right. \\
+g_{R} \rho_{\uparrow(\downarrow)}+\tilde{g}_{R} \rho_{\downarrow(\uparrow)} \pm \frac{g_{e} \mu_{B}}{2} \mathrm{~B}+g_{c}\left|\varphi_{\uparrow(\downarrow)}\right|^{2}+ \\
\left.+g_{x}\left|\varphi_{\downarrow(\uparrow)}\right|^{2}\right] \varphi_{\uparrow(\downarrow)}+\beta\left(\partial_{y} \pm i \partial_{x}\right) \varphi_{\downarrow(\uparrow)}, \\
\frac{\partial \rho_{\uparrow(\downarrow)}}{\partial t}=P_{\uparrow(\downarrow)}-\gamma_{R} \rho_{\uparrow(\downarrow)}-R \rho_{\uparrow(\downarrow)}\left|\varphi_{\uparrow(\downarrow)}\right|^{2},
\end{gathered}
$$

where $\nabla$ is nabla operator, $m_{\text {eff }}=\frac{2 m_{t} m_{l}}{m_{t}+m_{l}}$ is the effective mass of the polaritons, $m_{t}$ and $m_{l}$ is the transverse and longitudinal masses of the polaritons, $\gamma_{c}$ and $\gamma_{R}$ are the condensate and the reservoir dumping rates, $g_{c}$ and $g_{x}$ are coefficients of nonlinear interaction between the polaritons belonging to the same and to the orthogonal polarizations, $g_{R}$ and $\tilde{g}_{R}$ are the coefficients of the polariton-reservoir interactions. The magnetic field B shifts the eigrnfrequencies of the spin-up and spin-down polaritons in the opposite directions leading to the eigenfrequencies difference $g_{e} \mu_{B} \mathrm{~B}$ where where $g_{e}$ denotes the effective excitonic g-factor and $\mu_{B}$ is the Bohr magneton. The interaction between spin-up and spin-down components appearing because of TE-TM splitting is accounted by the coefficient $\beta$ which can be expresses via the transverse and longitudinal effective masses of the polartions $\beta=\frac{\hbar^{2}}{4}\left(\frac{1}{m_{l}}-\frac{t}{m_{l}}\right)$. The reservoir of incoherent polaritons is formed by an external pump with a spatially dependent particle generation rate $P$. The incoherent excitons relaxation rate is $\gamma_{R}$. The parameter of coupling between reservoir and condensate is denoted as $R$.

For theoretical consideration of the problem it is convenient to introduce dimensionless units. We normalize the time on the coherent polaritons relaxation rate $\gamma_{c}$, the spatial coordinate is normalized on characteristic length $L_{n}=\sqrt{\frac{\hbar}{m_{e f f} \gamma_{c}}}$. It is also convinient to introduce a dimensionless order parameter functions $\psi_{\uparrow(\downarrow)}=$ $\sqrt{\frac{g_{c}}{\hbar \gamma_{c}}} \varphi_{\uparrow(\downarrow)}$ and dimensionless exciton density $n_{\uparrow(\downarrow)}=$ $\frac{R}{2 \gamma_{c}} \rho_{\uparrow(\downarrow)}$. Then the equations (1),(2) can be written in the form

$$
\begin{array}{r}
i \partial_{t} \psi_{\uparrow}=\left[\frac{1}{2} \nabla^{2}+B+g n_{\uparrow}+\tilde{g} n_{\downarrow}+\left|\psi_{\uparrow}\right|^{2}+\right. \\
\left.+h\left|\psi_{\downarrow}\right|^{2}+i\left(n_{\uparrow}-\frac{1}{2}\right)\right] \psi_{\uparrow}+\sigma\left(\partial_{x}-i \partial_{y}\right)^{2} \psi_{\downarrow}, \\
\partial_{t} n_{\uparrow}=I_{\uparrow}-\left(\Gamma+G\left|\psi_{\uparrow}\right|^{2}\right) n_{\uparrow}, \\
i \partial_{t} \psi_{\downarrow}=\left[\frac{1}{2} \nabla^{2}-B+g n_{\downarrow}+\tilde{g} n_{\uparrow}+\left|\psi_{\downarrow}\right|^{2}+\right. \\
\left.+h\left|\psi_{\uparrow}\right|^{2}+i\left(n_{\downarrow}-\frac{1}{2}\right)\right] \psi_{\downarrow}+\sigma\left(\partial_{x}+i \partial_{y}\right)^{2} \psi_{\uparrow}, \\
\partial_{t} n_{\downarrow}=I_{\downarrow}-\left(\Gamma+G\left|\psi_{\downarrow}\right|^{2}\right) n_{\downarrow},
\end{array}
$$

where $B=\frac{g \mu_{B}}{2 \hbar \gamma_{c}} \mathrm{~B}$ is the dimensionless magnetic field, $g=\frac{2 g_{c}}{\hbar R}$ and $\tilde{g}=\frac{2 \tilde{g}_{c}}{\hbar R}$ account for the polariton condensate interaction with incoherent excitons of the same and the orthogonal polarizations, $h=\frac{g_{x}}{g_{c}}$ is the coefficient accounting for nonlinear cross polarization interaction, $\sigma=\frac{m_{t}-m_{l}}{m_{t}+m_{l}}$ is the strength of the TE-TM splitting (photonic effective spin-orbit interaction), $\Gamma=\frac{\gamma_{R}}{\gamma_{c}}$ is the decay rate of incoherent excitons, $G=\frac{\hbar R}{g_{c}}$ accounts for the depletion of the exciton reservoir due to the condensation into the coherent part, finally, $I_{\uparrow(\downarrow)}=\frac{R}{2 \gamma_{c}^{2}} P_{\uparrow(\downarrow)}$ are the dimensionless optical pumping powers in the right and the left circular polarizations.

The typical values of the system are the following: polariton mass $m_{\text {eff }}=10^{-4} m_{e}$, where $m_{e}$ is free electron mass; the coherent polaritons relaxation rate $\gamma_{c}=0.05$ $\mathrm{ps}^{-1}$; the stimulated scattering rate $R=0.01 \mu \mathrm{m}^{2} / \mathrm{ps}$; the effective nonlinearity (blue shift caused by the coherent polaritons) $g_{c}=6 \times 10^{-3} \mathrm{meV} \mu \mathrm{m}^{2}$. Thus the time is normalized on the characteristic time scale $\tau=1 / \Gamma_{c}=20 \mathrm{ps}$, the spatial coordinates are normalized 
on the length of $L=3.4 \mu \mathrm{m}$. All numerical simulations presented in this paper are obtained for the parameters $g=3.64, \tilde{g}=-0.364, h=-0.1, \sigma=0.03, \Gamma=3$ and $G=1.1$. The values of the coefficients are taken to reflect typical experimental conditions. In what follows we consider only the case of equal pumping power in both polarizations $I_{\uparrow}=I_{\downarrow}=I$.

\section{A. Formation of the vortices in zero magnetic field}

We consider the case of the radially symmetric annular pump, with a profile given by:

$$
I(r)=A_{I}\left[\exp \left(-\frac{r-R_{p}}{W_{p}}\right)+\exp \left(-\frac{r-R_{p}}{W_{p}}\right)\right]
$$

where $r$ is the radius, $A_{I}$ is the intensity of the optical incoherent pump, $R_{p}$ is the radius of the pump ring, $W_{p}$ is the width of the pump ring. In our numerical simulations these parameters were chosen to be $A_{I}=3.6$, $R_{p}=1.7$, and $W_{p}=1$. The spatial distribution of the pump is shown in Fig. 1. This pump creates a bath of incoherent excitons, which produces both trapping ring shape potential and positive gain for the coherent polariton condensate located in the center of the ring. Polariton condensation occurs when the exciton reservoir density exceeds a certain threshold value defined mostly by the losses in the system.
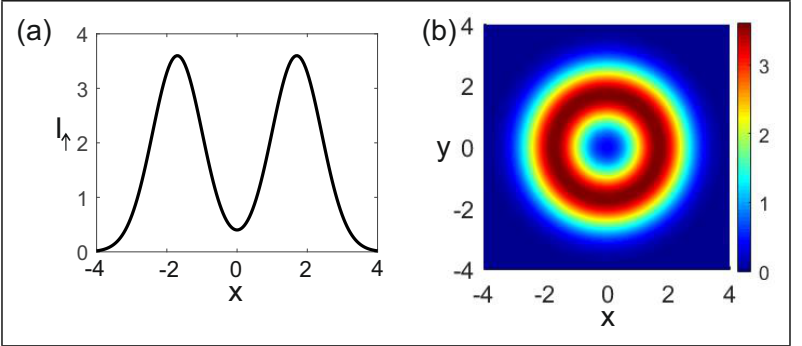

FIG. 1. The distribution of the intensity of the external pump is illustrated in panels (a) cross-section at $y=0$ (b) $2 \mathrm{D}$ colorplot. The distribution of the incoherent excitons $n$ has a similar structure corresponding to the ring-shaped profile.

The state of the condensate formed in the considered driven-dissipative model strongly depends on the size of the pumping spot. We chose the pumping parameters to ensure that only the states with vorticity \pm 1 in each spin component can be formed. In the following we refer to the states with positive and negative vorticity signs, corresponding to clockwise and anti-clockwise rotations, as to vortex and anti-vortex respectively. Let us mention that in the traps of smaller size only the states with zero vorticity can form. In the traps of larger radius the state with higher topological charges can form, see [37] for details. The effect of the magnetic field on the formation of the multi-vortex states in the presence of TE-TM splitting is an interesting problem but is out of the scope of the present paper and will be considered elsewhere.
As each of the two spin components is characterized by the topological charge \pm 1 , there are four possible states of the condensate. We thus denote the probability of formation of the state with vortices in both polarizations as $P_{+,+}$, with antivortices in both polarizations as $P_{-,-}$, with a vortex in the first and an antivortex in the second polarization - as $P_{+,-}$and the state with an antivortex in the first polarization and an antivortex in the second polarization as $P_{-,+}$.

To study the formation of the formation of stationary polariton states we performed a series of numerical experiments. For every run of the numerical simulations the initial conditions for both the polariton order parameter function and the incoherent exciton density were taken in the form of weak noise (so that all nonlinear terms are negligible at the initial stage of the condensate dynamics) with spatial correlation length much smaller compared to the characteristic sizes of the forming polariton and exciton components. For every iteration we observed formation of a stable stationary state and determined topological charges in both spin components. The statistics of the observed parameters over realizations with random initial conditions yields the probabilities of the formation of each of the four possible states.

As expected, in the absence of the polarization splittings the formation probabilities are the same and equal to $1 / 4$ for all the states. However, the presence of the effective spin-orbit coupling, provided by the TE-TM spitting of the photonic mode and Zeeman splitting produced by external magnetic field dramatically changes the statistics. We have checked that the obtained statistics is not sensitive to small variations of the intensity and the correlation length of the noise taken as the initial conditions.

Let us first consider the case of zero magnetic field $B=0$. For the chosen parameters in the presence of the spin-orbit coupling the probability of the formation of the stable state $(+,-)$ with the vortex in the right-hand and antivortex in the left-hand polarization, illustrated by the Fig. 2, exceeds 0.9. In this case the vortex and antivortex are phase-locked and thus the intenisties and the polarization of the resulting state do not depend on time. The second most probable stable state $(-,+)$, on the contrary, is characterized by an antivortex in the spin up component and a vortex in the spin down component. It is worth noting that the probabilities of the formation of symmetry breaking states with nonzero total vorticity (two vortices or two antivortices) was found to be very low (in 100 numerical experiments we did not register any of such events). Below it will be shown that different probabilities of the formations are explained by the fact that the states have different eienfrequencies. 


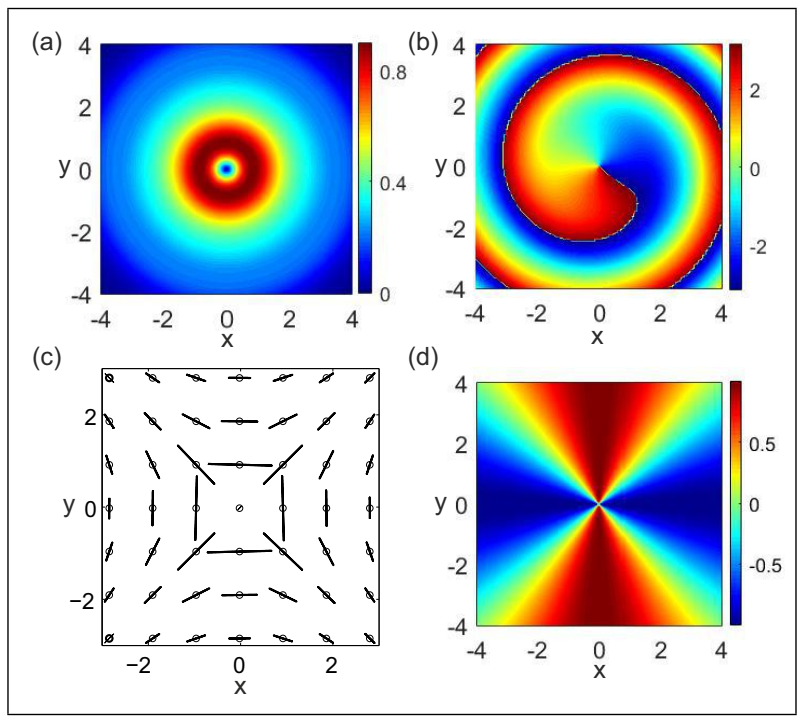

FIG. 2. The distributions of the density and the phase of a condensate in spin-up polarization is shown in panel (a) and (b) for zero magnetic field $B=0$ and incoherent pump amplitude $A_{I}=3.6$ exceeding the condensation threshold. The polarization ellipses are shown in panel (c) at the points marked by open circles. It is clearly seen that the polaritons are linearly polarized. To illustrate the polarization of the polariton field the Stokes parameters are calculated. The numerical simulations revealed that $S_{3}$ is very close to zero and so the optical field is linearly polarized. The distribution of $S_{1}$ parameter is shown in panel (d). The distribution of $S_{2}$ is the same as $S_{1}$ but rotated by the angle $\pi / 4$.

\section{B. The effect of the magnetic field on the vortex formation}

An important observation is that the introduction of the nonzero magnetic field does not affect the observed statistics much until the magnetic field exceeds a certain threshold. However, beyond this threshold the crossover takes place and quite surprisingly the state with two vortices of the same vorticity sign in both polarizations, $(+,+)$ or $(-,-)$, becomes dominant. The chosen sign of vorticity in this state, illustrated by the Fig. 3, depends on the direction of the magnetic field.

It is also worth mentioning that the frequencies of the coherent polariton emission in the two circular polarizations, which correspond to the energies of the two spin components, are different. The discrepancy in the frequencies produces beats and results in a periodically rotating polarization pattern in the orbitally stable state.

One can see that the field distributions in the two spin components are different and are asymmetric. As it is seen in Fig. 3 the spin-up component of $(+,+)$ state is much more symmetric compared to its spin-down counterpart. This can be explained by the fact that the vortex in the spin-up polarization is coupled to a linear mode with negative angular momentum -1 in the spin-down polarization efficiently by the TE-TM field. However the

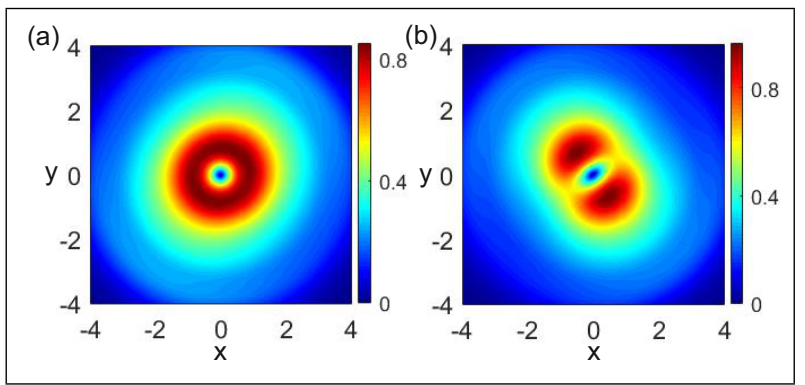

FIG. 3. The distributions of the densities of the condensates in up and down spin components for $(+,+)$ state are shown in panels (a) and (b) correspondingly. Magnetic field $B=0.2$, the incoherent pump $A_{I}=3.6$ exceeds the threshold value and thus the condensation takes place. It is seen that the density distributions are not radially symmetric, this particularly applies to the density of the spin-down component. The shape of the state is constant, but rotates as a whole with the frequency equal to the detuning of the spin-up polarization frequency from the frequency of the spin-down state.

spin-orbit interaction, stemming from the TE-TM splitting of the photonic mode, couples a vortex in the spindown polarization to a mode with topological charge +3 in the spin-up component. The eigenfrequency of this mode is far detuned from the frequency of the vortex and thus the excitation is inefficient. So the field in the spin-up component is nearly a pure vortex but the field of the spin-down component is a combination of a vortex and a mode with the opposite angular momentum and low amplitude. Strong magnetic field supresses the excitation of the quasi-linear mode in the spin-down state by the vortex in the spin-up state and so in strong magnetic field the state becomes symmetric.

The time evolution of the polarization of the optical field is illustrated in Fig. 4. As it is seen in panel (a) all three Stokes parameters are oscillating and the polarization is not linear at all times. In panels (b)-(k) the spatial distributions of the Stokes parameters at different times are shown. It is seen that the optical field is elliptically polarized and the polarization ellipse rotates with time. The orientation of the polarization ellipse at a given time does not depend much on the observation point.

If the magnetic field is decreased after the formation of $(+,+)$ state, this state remains stable, but no synchronization of the spin-up and the spin-down components of the state was observed in our numerical simulations. This can be explained by the absence of coupling of the vortices in the two spin components by the TE-TM splitting. Such a coupling can appear if the angular symmetry of the vortex and the anti-vortex is broken, but this effect was not observed in our simulations for the chosen sets of the parameters.

It is worth making a remark about $(+,-)$ state. If we let this state form in a low magnetic field then the increase of the magnetic field above the threshold value breaks the synchronization, spin-up and the spin-down components desynchronize and split in frequency, and 


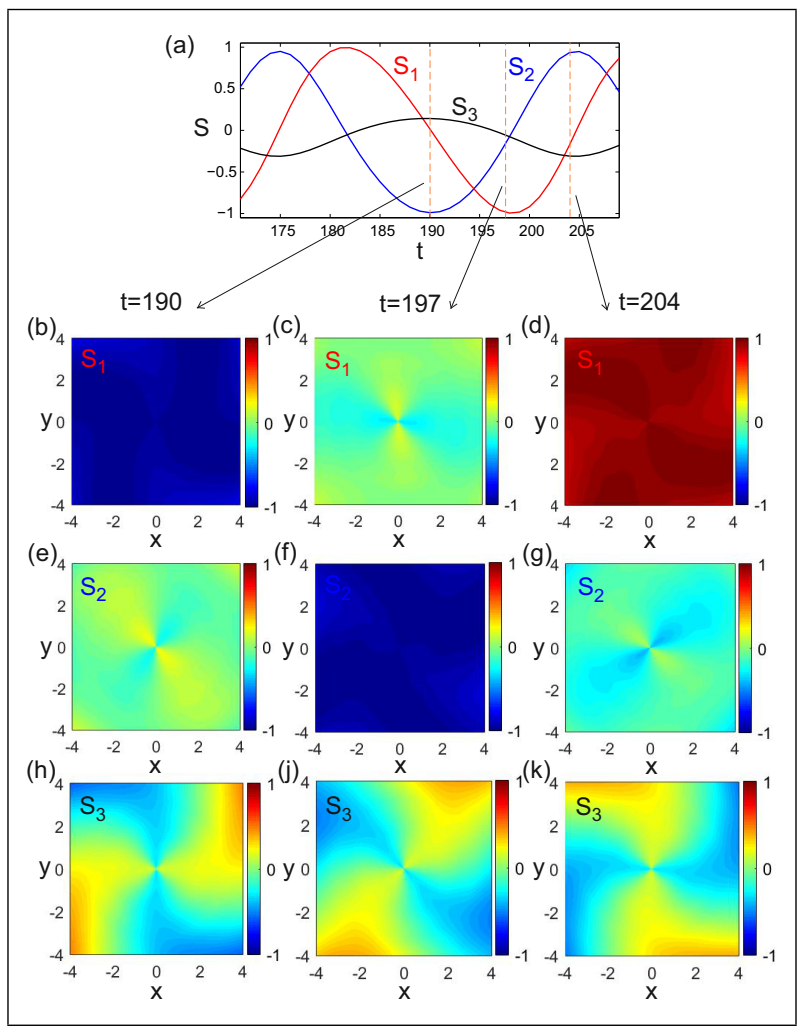

FIG. 4. The dynamics of the Stokes parameters for $(+,+)$ state is shown for the polaritons places in magnetic field $B=0.2$. The normalized Stokes parameters $S_{1}, S_{2}$ and $S_{3}$ measured at $x=0, y=0.85$ are shown in panel (a) as functions of time. The spatial distributions of $S_{1}$ are shown in (b)-(d) for $t=190, t=197$ and $t=204$ correspondingly. The distributions of $S_{2}$ and $S_{3}$ at the same moments of time are shown in panels (e)-(g) and (h)-(k). It is seen that the optical field is elliptically polarized and that the ellipticity changes in time periodically. It is also seen that the orientation of the polarization ellipse does not depend on the coordinates strongly.

stationary state $(+,-)$ is transformed to an oscillating one.

The probabilities of the formation of the four possible states are plotted as functions of the magnetic field in Fig. 5. The measured quantities characterizing the condensates are averaged over 100 numerical solutions of Eqs. (3-6), simulating condensates excited by the incoherent pump. The initial conditions for the simulations are taken in the form of a weak noise in the photonic and the excitonic components. The dependence of the averaged topological charge in both spin components $m_{1}$ and $m_{2}$, as well as the total averaged topological charge defined as $m_{t}=\left(m_{1}+m_{2}\right) / 2$, on the magnetic field is shown in Fig. 5b. The averaged topological charges in the two spin components have different signs at low magnetic fields below the threshold, making total topological charge zero. On the contrary, above the threshold the average topological charge in the second polarization switches sign, and average total topological charge rapidly reaches values close to 0.8 . This means that the application of the magnetic field leads to the spinning of the polariton condensate, which is nontrivial effect, as polaritons are electrically neutral particles and thus their orbital motion is not affected directly by the magnetic field.

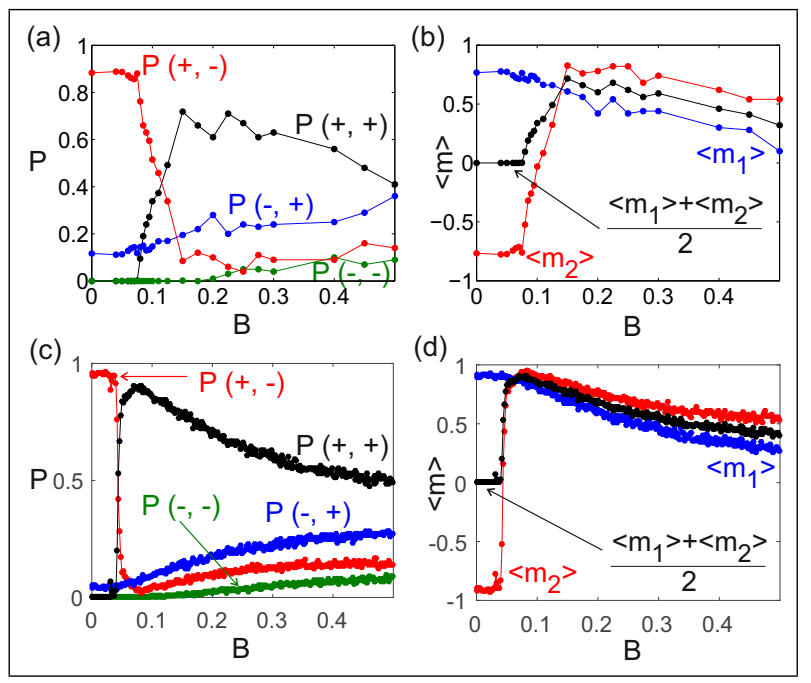

FIG. 5. Influence of the magnetic field $B$ on statistics of the forming vortex states. Panels (a) and (b) correspond to the full 2D model given by Eqs.3-6, while panels (c) and (d)- to simplified toy model described by Eqs.9-16. Panels (a) and (c) show the probabilities of the formation of each of the four possible stable states. Panels (b) and (d) show mean topological charges. The averaging is done over 100 simulations for panels (a),(b) and over 1000 simulations for panels (c),(d).

\section{DISCUSSION OF THE NUMERICAL RESULTS}

To understand the reported effects we propose a simple hypothesis that the winning state inherits the properties of the mode which reaches the regime of gain saturation first. As it is mentioned above, normally this is the fastest growing mode. However, choosing the appropriate initial conditions it is possible to make a mode with non-maximal growth rate win. This explains that in the numerical simulations different modes win with different probabilities. However in the case of the initial conditions in the form of a weak noise the fastest growing mode has the highest probability of winning the competition.

In the following we represent the spinor condensate wavefunction as the series $\psi_{\uparrow(\downarrow)}=\sum_{m=-\infty}^{\infty} \psi_{\uparrow(\downarrow), m}$ with $\psi_{\uparrow(\downarrow), m}=\rho_{\uparrow(\downarrow), m}(r) \exp (i m \theta)$, where $\rho_{\uparrow(\downarrow), m}$ are functions of radial coordinate $r, \theta$ is the angular coordinate, and $m$ is the integer orbital index of the corresponding mode. For the chosen set of parameters the results of the full 2D numerical simulations allow us to neglect all modes for which $|l| \neq 1$ in any of the spin components. Thus the state of a condensate can be represented in the 
spinor form:

$$
\begin{aligned}
\Psi=\left(\begin{array}{c}
\psi_{\uparrow} \\
\psi_{\downarrow}
\end{array}\right)= & {\left[C_{\uparrow+}\left(\begin{array}{c}
e^{i \theta} \\
0
\end{array}\right)+C_{\uparrow-}\left(\begin{array}{c}
e^{-i \theta} \\
0
\end{array}\right)+\right.} \\
& \left.+C_{\downarrow+}\left(\begin{array}{c}
0 \\
e^{i \theta}
\end{array}\right)+C_{\downarrow-}\left(\begin{array}{c}
0 \\
e^{-i \theta}
\end{array}\right)\right] g(r)
\end{aligned}
$$

where $C_{\uparrow+}, C_{\uparrow_{-}}, C_{\downarrow+}, C_{\downarrow-}$ are the complex amplitudes of the components corresponding to the vortex and antivortex in the spin up and the spin-down component. The function $g(r)$ describes the radial structure of the mode and is normalized to unity.

It is instructive to introduce such quantities as the number of particles $N_{\uparrow(\downarrow)}=\int|\psi \uparrow(\downarrow)|^{2} d x d y$ and the momentum $M_{\uparrow(\downarrow)}=\int \psi_{\uparrow(\downarrow)}^{*} \hat{L}_{a m} \psi_{\uparrow(\downarrow)} d x d y$,

$$
\hat{L}_{a m}=-i\left(x \partial_{y}-y \partial_{x}\right)
$$

in each of the polarization. It is straightforward to show that, under the assumption that the contribution of the excitations with the angular momentum $l \neq \pm 1$ can be neglected, the number of particles can be expressed as $N_{\uparrow(\downarrow)}=\left|C_{\uparrow(\downarrow)+}\right|^{2}+\left|C_{\uparrow(\downarrow)-}\right|^{2}$ and the angular momentum as $M_{\uparrow(\downarrow)}=\left|C_{\uparrow(\downarrow)+}\right|^{2}-\left|C_{\uparrow(\downarrow)-}\right|^{2}$. The present paper addresses exactly this case and it has been checked by direct numerical simulations that the assumption is valid for the chosen range of the parameters. Let us note that in the considered case the squared amplitudes $\left|C_{\uparrow(\downarrow) \pm}\right|^{2}$ can be expressed in the measured particles numbers and the momenta $\left|C_{\uparrow(\downarrow) \pm}\right|^{2}=\frac{1}{2}\left(N_{\uparrow(\downarrow)} \pm M_{\uparrow(\downarrow)}\right)$.

The behaviour of the number of particles and the momenta of each of the polarizations are presented in panels (a) and (b) of Fig. 6 by the solid lines. The simulations are performed for the case when the magnetic field is absent $B=0$. The amplitudes of the components $\left|C_{\uparrow(\downarrow) \pm}\right|^{2}$ are shown in panels (c) and (d).

It is seen that the numbers of the particles grow from the noise in both polarizations and after some time reach steady states. It is worth noting that initially, when the density of the coherent polaritons is relatively low, the momenta are close to zero. Indeed, the density of the polaritons starts to deviate noticeably from zero at $t \approx 50$. The angular momentum in this regime stays low in both polarizations. Correspondingly, the amplitudes of the excitations with $l=1$ and $l=-1$ are equal to each other in both polarizations, see panels (c) and (d). However, at $t \approx 70$ the angular momentum starts to grow. The dependencies of the amplitudes on time show that this is happening because the excitation with $l=-1$ gets suppressed in the spin-up polarization and the excitation with $l=1$ gets suppressed in the spin-down polarization. Thus the competition between the excitaions with different vorticities is the mechanism of the steady state formation.

To explain the competition between the excitations we consider the structure of the eigenmodes of the system. Let us mention an important fact that in the presence of the TE-TM splitting none of the vectors $\left(e^{ \pm i \theta}, 0\right)^{\mathrm{T}}$,
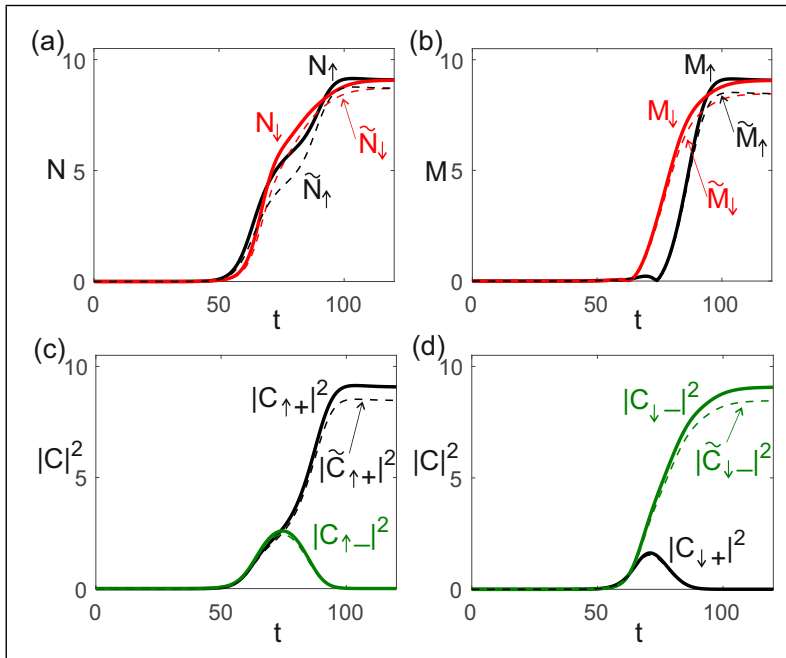

FIG. 6. The temporal dynamics of the numbers of particles and the angular momenta obtained from direct numerical modelling of equations (3)-(6) are shown in panels (a),(b) by solid lines marked as $N_{\uparrow(\downarrow)}$ and $M_{\uparrow(\downarrow)}$. The number of particles and the momentum are defined as $N_{\uparrow(\downarrow) \pm}=$ $\int|\psi \uparrow(\downarrow)|^{2} d x d y$ and $M_{\uparrow(\downarrow) \pm}=\int \psi_{\uparrow(\downarrow)}^{*} \hat{L}_{a m} \psi_{\uparrow(\downarrow)} d x d y, \hat{L}_{a m}=$ $-i\left(x \partial_{y}-y \partial_{x}\right)$. The dashed lines in panels (a),(b) show the dynamics of the particle number $\tilde{N}_{\uparrow(\downarrow)}=\left|\tilde{C}_{\uparrow(\downarrow)+}\right|^{2}+\left|\tilde{C}_{\uparrow(\downarrow)-}\right|^{2}$ and the angular momentum $\tilde{M}_{\uparrow(\downarrow)}=\left|\tilde{C}_{\uparrow(\downarrow)+}\right|^{2}-\left|\tilde{C}_{\uparrow(\downarrow)-}\right|^{2}$ obtained from the simulation of the toy model (9)-(16). The behaviour of the squared amplitudes calculated as $\left|C_{\uparrow(\downarrow) \pm}\right|^{2}=$ $\left(N_{\uparrow(\downarrow)} \pm M_{\uparrow(\downarrow)}\right) / 2$ are shown by the solid lines marked as $C_{\uparrow(\downarrow) \pm}$ in panels (c),(d). These amplitudes can be considered as the amplitudes of the excitations $\left(e^{ \pm i \theta}, 0\right)^{T},\left(0, e^{ \pm i \theta}\right)^{T}$ provided that the contribution of the excitations with the other angular momenta are negligible. The dashed lines marked as $\tilde{C}_{\uparrow(\downarrow) \pm}$ in panels (c),(d) show behaviour of the amplitudes calculated from the toy model (9)-(16). In the simulation the magnetic field is absent $B=0$.

$\left(0, e^{ \pm i \theta}\right)^{\mathrm{T}}$ corresponds to an eigenmode. However, below we will argue that the modes $g\left(e^{-i \theta}, 0\right)^{\mathrm{T}}, g\left(0, e^{i \theta}\right)^{\mathrm{T}}$ are close to the eigenstates. This is so because TE-TM splitting couples these modes only to the excitations with topological charge equal to \pm 3 that are not supported by the effective potential created by the chosen incoherent pump and thus the components $l= \pm 3$ are small.

In contrast, the TE-TM splitting couples a vortex in the spin-up polarization with an antivortex in the spindown polarization, provided that the magnetic field is suffiently low, so that the Zeeman splitting desynchronizes the two spin components. In this case the eigenstates of the systems can be approximated in the form of the mixed states

$$
\frac{g}{\sqrt{a_{1}^{2}+a_{2}^{2}}}\left[a_{1}\left(\begin{array}{c}
e^{i \theta} \\
0
\end{array}\right) \pm a_{2}\left(\begin{array}{c}
0 \\
e^{-i \theta}
\end{array}\right)\right],
$$

where the complex coefficients $a_{1,2}$ account for the mutual phases of the spin-up and spin-down polarizations and for the difference in the particles numbers in the polarizations. 
In zero magnetic field the mixed eigenstates are $\frac{g}{\sqrt{2}}\left(e^{i \theta}, e^{-i \theta}\right)^{T}$ and $\frac{g}{\sqrt{2}}\left(e^{i \theta},-e^{-i \theta}\right)^{\mathrm{T}}$, reflecting the system symmetry. The amplitudes of these modes $D_{1}$ and $D_{2}$ can be expressed in the amplitudes $C_{\uparrow,-}$ and $C_{\downarrow,+}$ as $D_{1}=\frac{1}{\sqrt{2}}\left(C_{\uparrow+}+C_{\downarrow-}\right)$ and $D_{2}=\frac{1}{\sqrt{2}}\left(C_{\uparrow+}-C_{\downarrow-}\right)$.

The dynamics of the amplitudes is shown in panel (a) of Fig. 7 in the logarithmic scale. Note that because of the effective gain the amplitudes $\left|C_{1-}\right|$ and $\left|C_{2+}\right|$ grow exponentially in the linear regime. However, this is not so for the amplitudes $\left|C_{1+}\right|$ and $\left|C_{2-}\right|$, as the states $\left(0, e^{i \theta}\right)^{T},\left(e^{-i \theta}, 0\right)^{T}$ are close to the eigenmodes of the system, in contrast to the states $\left(e^{i \theta}, 0\right)^{T},\left(0, e^{-i \theta}\right)^{T}$. At the same time, the dependence of the amplitudes of the mixed states $D_{1}$ and $D_{2}$ show the exponential growth in the linear regime. This allows us to conclude that the mixed states $\frac{1}{\sqrt{2}}\left(e^{i \theta}, \pm e^{-i \theta}\right)^{T}$ with a vortex in the spinup and an antivortex in the spin-down components are the eigenstates.

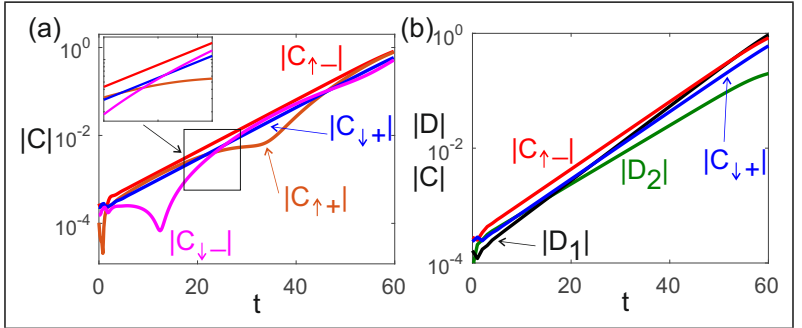

FIG. 7. The temporal dynamics of the amplitudes $C_{\uparrow(\downarrow), \pm}$ of the components $\left(e^{ \pm i \theta}, 0\right)^{T}$ and $\left(0, e^{-i \theta}\right)^{T}$ is shown in panel (a). It is seen that the amplitudes $C_{\uparrow,-}$ and $C_{\downarrow,+}$ grow exponentially in time (the vertical axis is in logarithmic scale) which is the indication that the the states $\left(0, e^{i \theta}\right)^{T},\left(e^{-i \theta}, 0\right)^{T}$ approximate the eigenstates of the system well. At the same time, it is seen that there are beatings between the amplitudes $C_{\uparrow,+}$ and $C_{\downarrow},-$ and therefore $\left(e^{i \theta}, 0\right)^{T}$ and $\left(0, e^{-i \theta}\right)^{T}$ are not the eigenstates of the system. To prove that the mixed states $\frac{1}{\sqrt{2}}\left(e^{i \theta}, \pm e^{-i \theta}\right)^{T}$ are the eigenmodes the dependencies of the amplitudes $D_{1,2}$ of these modes are shown in panel (b) as functions on time alongside with the dependencies of the amplitudes $C_{\uparrow,-}$ and $C_{\downarrow,+}$. It is seen that they all grow exponentially but with slightly different growth rates. This is the indication that they are the amplitudes of the eigenstates of the system. The simulations are performed for the case when the magnetic field is absent $B=0$.

We would like to highlight that the "mixed" mode with amplitude $D_{1}$ is growing with the fastest rate, and the other "mixed" mode $D_{2}$ has the slowest growing rate, the one-component modes having approximately the same growth rates. To explain this we propose a simple hypothesis. Let us remind an important fact that the exciton bath creates an effective potential with finite height and width, allowing the condensate polaritons to escape the trap, which produces the mechanism of the condensate decay stemming from tunneling outside of the trap. The corresponding contribution to the decay rate de- pends on the confinement energy of a particular state, being more pronounced for higher energy levels. Assuming that the gain and the radiative losses are universal for all the modes one can conclude that the modes with higher frequencies should experience higher losses. Let us remark that here by radiative losses we mean not the escape of the photons from the cavity but the escape of the coherent polaritons from the area of the effective gain produced by the incoherent excitons. From the point of view of the formation of the localized states this process can be considered as additional losses.

The coupling between the vortex in the spin-up component and an antivortex in the spin-down component results in the splitting of the frequencies of the mixed modes $D_{1}$ and $D_{2}$. The energies of the modes $C_{\uparrow-}$ and $C_{\downarrow+}$ are not affected by the spin-orbit interaction and are degenerate in accordance with the symmetry of the system. Therefore the first mixed mode $D_{1}$ is redshifted and is characterized with the lowest frequency of emission and decay rate, while the second mode mode $D_{2}$ has the highest frequency and the highest losses, which is schematically illustrated in Fig. 8.

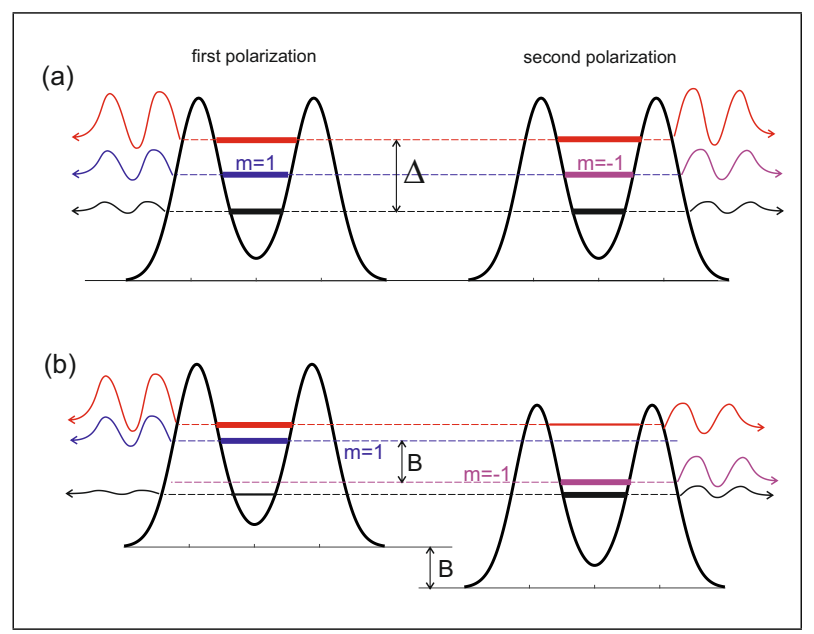

FIG. 8. The schematic picture illustrating the proposed hypothesis explaining the observed polariton dynamics and modes selection. The effective potentials for the polaritons is the first and in the second polaizations are shown in the left and in the right columns correspondingly. The blue and magenta horizontal lines show the energy levels of the polaritons without spin-orbit interaction (without TE-TM splitting). The red and the black horizontal lines show the energy levels of the hybrid eigenstates that have components of both polarizations because of the spin-orbit interaction resulting in the energy splitting $\Delta$. The thickness of these lines schematically shows the portion of the component in the hybrid mode. The upper row illustrate the case without and the lower row - with magnetic field $B$. The oscillating lines denote the radiation appearing because of the tunneling of the polaritons through the potential barrier of finite width and height. The amplitude of the oscillations of the lines shows the intensity of the tunneling and, correspondingly, the radiative losses of the mode.

We can conclude that in the absence of a magnetic field 
the fastest growing and subsequently the winning mode is $D_{1}$, as can be seen in Fig. 9 showing the dependence of the amplitudes of the eigenmodes in linear scale for times long enough for the formation of a stable stationary vortex state. Both components of the winning mixed mode at zero magnetic field have the same absolute values and thus the growth of this mode results in the establishment of the state with a vortex in the spin-up component and an antivortex in the spin-down component. The growth of the other eigenmodes gets suppressed when the density of the polariton condensates becomes high enough. It is good to notice here that in the case of the resonant pump this effect is not possible because in this case the frequency of the state is fixed by the pump and thus frequency dependent radiative losses cannot be the mechanizm responsible for the selection of the winning state.

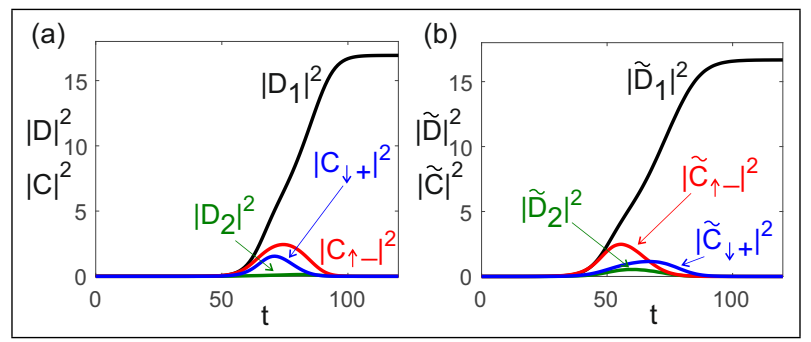

FIG. 9. The dynamics of the amplitudes of the eigenmodes in the linear scale. Panel (a) is for full 2D simulations, panel (b) - for the toy model. Comparing the panels one can conclude that the toy model reproduces the results of numerical simulations of the full $2 \mathrm{D}$ model well enough. The magnetic field is absent $B=0$.

We now switch to the case of the relatively strong magnetic fields, where another stationary state wins the mode competition. Let us take into account that the magnetic field lifts the degeneracy, shifting the balance of the components $C_{\uparrow+}$ and $C_{\downarrow-}$ - within the fastest growing mixed mode $D_{1}$. In the limit of strong magnetic field one of the components dominates the other, so in the stable regime, where the growth of the mode is saturated, it cannot provide the formation of an antivortex in the spin-down component. The antivortex in the spin-down component can potentially be produced by the second mixed mode, but its growth rate is the lowest. Then it is reasonable to expect that the final state in the second polarization is produced by the second fastest growing mode having nonzero component in the second polarization $C_{\downarrow+}$ which means that the angular momentum in the spin-down polarization has to be $l=1$. The vortices form in both of the polarizations.

The dynamics of the angular momenta is shown in Fig. 10. It is worth mentioning that the absolute value of the angular momentum of the spin-up component is higher than that of the spin-down component. Indeed, as it is seen from the panels, showing the dynamics of the amplitudes, the amplitude $C_{\downarrow-}$ does not decay to zero but stays at some relatively low level. Since the angular momentum in the spin-down component is
$M_{\downarrow}=\left|C_{\downarrow+}\right|^{2}-\left|C_{\downarrow-}\right|^{2}$ the nonzero $C_{\downarrow-}$ reduces the angular momentum in the spin-down component. The nonzero value of $C_{\downarrow-}$ is the direct consequence of scattering from the vortex in the spin-up component due to the spin-orbit coupling.
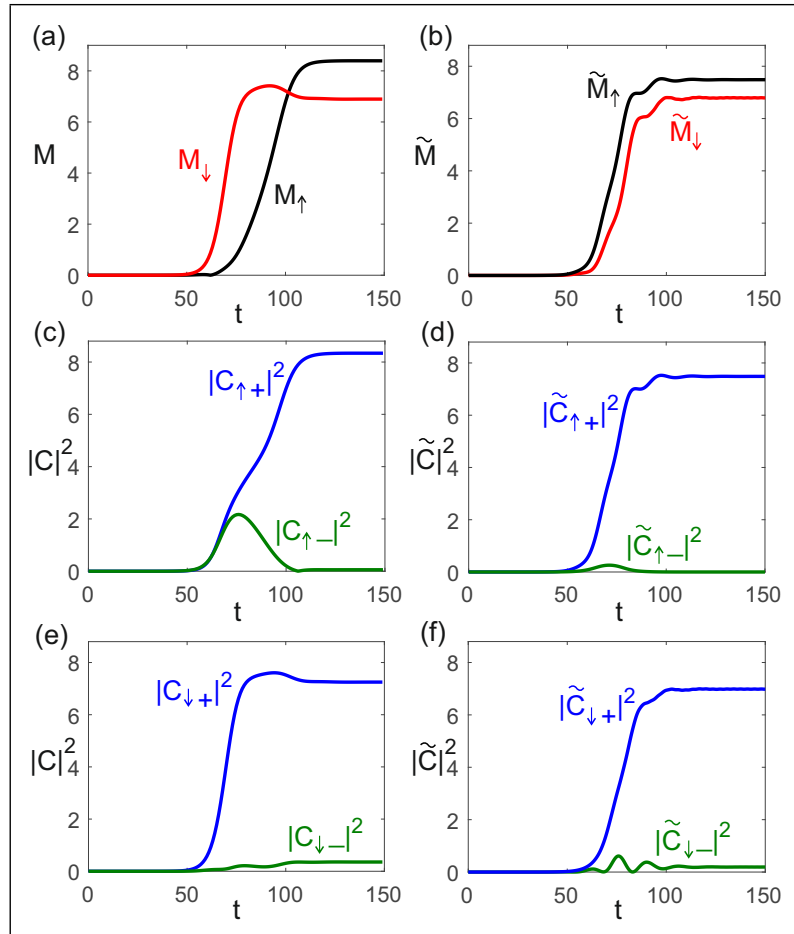

FIG. 10. The temporal dynamics of the particle number and the momenta of the fields in the first and the second polarization. panel (a) is for 2D simulations, (b) is for the toy model. The dynamics of the components $|C|^{2}$ are shown in panels (c)-(f). Panels (c) and (e) are for 2D simulations, (d) and (f) are for the toy model. It is seen that the results of the modeling of full $2 \mathrm{D}$ equations complies very well with the results obtained by the toy model. The magnetic field $B=0.2$.

To summarize, we can conclude that for stronger magnetic fields we should observe the formation of the vortices in both spin components. Naturally, inversing the sign of the magnetic field it is possible to get antivortices in both spin components.

\section{A. The toy model}

To illustrate the proposed hypothesis we develop a toy model based on a system of the rate equations mimicking the dynamics of the initial two-dimensional system. Our model consists of four oscillators that are characterized by their complex amplitudes $\tilde{C}_{\uparrow(\downarrow) \pm}$ describing the components with $l= \pm 1$ angular momentum in each of the polarizations. As it was discussed before only two of these oscillators interact linearly because of the spin-orbit interaction, these are the oscillators $\tilde{C}_{\uparrow+}$ and $\tilde{C}_{\downarrow-}$. However, all oscillators belonging to the same polarization 
interact to each other nonlinearly through the depletion of the common effective gain. This allows to write the toy model for the oscillators in the form

$$
\begin{array}{r}
\partial_{t} \tilde{C}_{\uparrow+}=\left(-\gamma_{1}+i B-\beta\left(\left|\tilde{C}_{\uparrow+}\right|^{2}+\right.\right. \\
\left.\left.+2\left|\tilde{C}_{\uparrow-}\right|^{2}\right)\right) \tilde{C}_{\uparrow+}+i \sigma_{e f f} \tilde{C}_{\downarrow-}+\mu_{+} \\
\partial_{t} \tilde{C}_{\uparrow-}=\left(-\gamma_{1}+i B-\beta\left(\left|\tilde{C}_{\uparrow-}\right|^{2}+\right.\right. \\
\left.\left.+2\left|\tilde{C}_{\uparrow+}\right|^{2}\right)\right) \tilde{C}_{\uparrow-}+\mu_{-} \\
\partial_{t} \tilde{C}_{\downarrow+}=\left(-\gamma_{1}-i B-\beta\left(\left|\tilde{C}_{\downarrow+}\right|^{2}+\right.\right. \\
\left.\left.+2\left|\tilde{C}_{\downarrow-}\right|^{2}\right)\right) \tilde{C}_{\downarrow+}+\eta_{+} \\
\partial_{t} \tilde{C}_{\downarrow-}=\left(-\gamma_{1}-i B-\beta\left(\left|\tilde{C}_{\downarrow-}\right|^{2}+\right.\right. \\
\left.\left.+2\left|\tilde{C}_{\downarrow+}\right|^{2}\right)\right) \tilde{C}_{\downarrow-}+i \sigma_{e f f} \tilde{C}_{\uparrow+}+\eta_{-}
\end{array}
$$

The linear losses and gain are proportional to $\gamma_{1}\left(\gamma_{1}<0\right.$ corresponds to gain). The nonlinear losses (depletion of the gain) for each polarization depends only on the amplitudes of the modes belonging to the same polarization. The nonlinear term has the standard form containing the components with different angular momenta. Let us mention here that such a nonlinear term provides competition between the modes and the stronger mode suppresses the weaker one. The terms proportional to the magnetic field $B$ account for the Zeeman splitting.

Now we need to add a key ingredient, the frequency dependent losses. The real dependencies of the radiative losses on frequency is difficult to reproduce by a simple model and thus we utilize a simplest way to realize such losses - we couple each of the amplitude $\tilde{C}_{\uparrow(\downarrow) \pm}$ to a detuned linear oscillator with losses. We stress that this part of the model is completely phenomenological and we fail to propose any microscopic justification for it. However, it reproduces well the results of the direct numerical simulations. The equations (13)-(16) for the amplitudes $\tilde{C}_{\uparrow(\downarrow) \pm}$ should be thus coupled to the equations for the auxiliary oscillators $\mu_{ \pm}, \eta_{ \pm}$having the form:

$$
\begin{aligned}
\partial_{t} \mu_{+} & =\left(-\gamma_{2}+i(\Omega+B)\right) \mu_{+}+i \alpha \tilde{C}_{\uparrow+} \\
\partial_{t} \mu_{-} & =\left(-\gamma_{2}+i(\Omega+B)\right) \mu_{-}+i \alpha \tilde{C}_{\uparrow-} \\
\partial_{t} \eta_{+} & =\left(-\gamma_{2}+i(\Omega-B)\right) \eta_{+}+i \alpha \tilde{C}_{\downarrow+} \\
\partial_{t} \eta_{-} & =\left(-\gamma_{2}+i(\Omega-B)\right) \eta_{-}+i \alpha \tilde{C}_{\downarrow-}
\end{aligned}
$$

Here $\gamma_{2}$ accounts for the linear losses in the detuned oscillators, $\Omega$ is the detuning and $\alpha$ is the coupling strength.

The losses introduced by the coupling to this oscillators depend on the frequency detuning of the modes from the corresponding "auxiliary" oscillators. It is important to mention that in the initial model the losses must not depend on the magnetic field in the absence of the spinorbit coupling. This is natural because the magnetic field shifts equally the energy of free propagating and trapped polaritons, so the probability of the tunneling through the barrier leading to the additional condensate losses does not change. This means that in our toy model the resonant frequency of the "auxiliary" resonators and the resonant frequency of the modes in the same polarization must depend on the magnetic field $B$ in the same way.
The coefficients of the toy model giving best fit of the results of the $2 \mathrm{D}$ numerical simulation are: $\gamma_{1}=0.55$, $\beta=0.01, \sigma_{\text {eff }}=0.06, \gamma_{2}=0.5, \Omega=2$ and $\alpha=0.9$.

The dependencies of the real and the imaginary parts of the frequencies of the eigenmodes are shown in panels (a) and (b) of Fig. 11 respectively. It is crucial that the interaction between the oscillators $\tilde{C}_{\uparrow+}$ and $\tilde{C}_{\downarrow-}$ leads to the splitting of the resonant frequencies and thus one of the mixed modes having higher frequency is coupled to auxiliary lossy oscillators stronger then the other mode having lower frequency. That is why the first mode experiences higher losses. Formally, this follows from the expression of the imaginary part of the frequency, see Fig. 11 showing the dependencies of the frequencies on the magnetic field value. The splitting of the modes decreases with magnetic field and therefore the difference in the growth rates decreases too. Note that the fastest growing mode is the mixed mode $D_{1}$, while the slowest growing mode is the other mixed mode $D_{2}$.
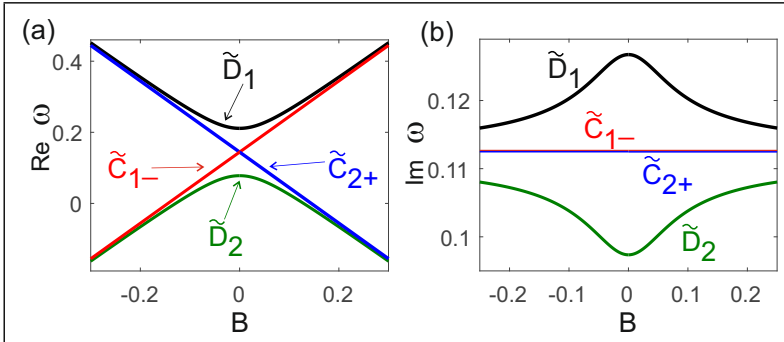

FIG. 11. The dependencies of the real (a) and imaginary (b) parts of the frequencies of the eigenmodes of the toy model as functions of the applied magnetic field $B$. It is seen that the coupling between $\tilde{C}_{\uparrow+}$ and $\tilde{C}_{\downarrow}$ - results in the formation of the mixed modes having different real and, what is more important for the discussed effects, imaginary parts. The modes $\tilde{C}_{\uparrow-}$ and $\tilde{C}_{\downarrow+}$ do not interact to other polariton modes and thus they have the same frequencies and losses that do not depend on the magnetic field. The parameters of the toy model are given in the text.

The number of particles and the angular momenta calculated with the toy model are shown in Fig. 6(a),(d) by dashed lines. It is seen that they are in good agreement with the $2 \mathrm{D}$ numerical simulations. Good agreement is also achieved for the dynamics of the amplitudes of the components and the dynamics of the eigenmodes (see panel (b) in Fig. 9). Note that, as expected from Fig. 11, the fastest growing and therefore the winning mode is the mixed mode $D_{1}$ which ensures the formation of the vortex in the spin-up component and antivortex in the spin-down component. The single component modes $C_{\uparrow-}$ and $C_{\downarrow+}$ in turn have the same growth rates that are lower then the increment of $D_{1}$ mixed mode but higher then the increment of $D_{2}$ mixed mode.

The example of the temporal dynamics of the toy model for magnetic field $B=0.2$, whic is above the crossover threshold is shown in Fig. 10(b),(d),(f) showing the evolutions of the angular momenta in spin-up and 
spin-down polarizations and the dynamics of the amplitudes of the modes. One may note that the results qualitatively reproduce those of the direct numerical simulations of full scale 2D model. One can see that the angular momentum in the spin-down component is positive as in the spin-up component. But the spin-down component also contains the an antivortex part, which manifests itself in the braking of the rotation symmetry visible in the panel (b) of Fig 3.

Finally, we did numerical experiment starting simulations from random noise and counting the probability of the formation of different states. The dependencies of the probabilities and the averaged angular momenta are shown in panels (c) and (d) of Fig. 5. The crossover is clearly seen and the results are in good agreement with the data obtained from the $2 \mathrm{D}$ modeling.

It is worth noting here that it is important that the magnetic field affect not only the growth rate of the linear modes but also their polarization structures. In the week magnetic field the fastest growing mode have approximately the same number of polaritons in the spin-up and the spin-down components and so both polarizations of the stationary state are defined by the structure of the mode. But the magnetic field affects the relative portions of the polaritons in the spin-up and the spin down components of the modes. So in relatively strong magnetic field most of the polaritons in the fastest growing mode belong either to spin-up or, depending on the sign of the magnetic field, to spin-down polarization. This is why when the growth of the fastest mode becomes saturated by the nonlinear effects the density of polaritons in one of the polarizations is still small and other modes continue to grow. Thus if the magnetic field exceeds the threshold then the polarization structure of the most probable stationary state is defined by the structures of the fastest and the second fastest growing modes. This explains the existence of the cross-over observed in the numerical simulations.

Let us note that both the results of the full $2 \mathrm{D}$ simula- tions and the results obtained with the toy model show that at strong magnetic fields the probabilities of the formation of different states tend to become equal. This can be understood from the dependencies of the real and imaginary parts of the eigenfrequencies on the magnetic field, shown in Fig. 11. Note, that extremely strong magnetic field suppresses the intermode coupling, so both the real and the imaginary parts of the eigenfrequencies become equal. Therefore, in very strong magnetic fields all modes have practically the same growth rates and all states should form with approximately the same probability. This agrees well with the statistics obtained from the numerical simulations. Therefore, there exists the value of a magnetic field, which is optimal for the achievement of the effect of the spinning.

\section{CONCLUSION}

In conclusion, we observed that cumulative effect of photonic spin-orbit coupling and excitonic Zeeman splitting results in the selection of the preferable sign of the angular momentum of the polariton condensates created by incoherent pump. This opens the way for the magnetic field control of the topological charges in a system composed of electrically neutral bosons. At low magnetic fields a condensate contains a vortex in one polarization and an antivortex in the other polarization. However, at some threshold value of the magnetic field a crossover occurs and depending on the sign of the magnetic field either vortices or antivortices form in both polarizations.

This work was supported by megagrant 14.Y26.31.0015 and Goszadanie no. 3.8884.2017/8.9 of the Ministry of Education and Science of Russian Federation. A.V.Y. and I.A.S. acknowledge support from the from the Icelandic Research Fund, Grant No. 163082-051. A.N. acknowledges support from RFBR Grant No. 18-32-00434 and from Icelandic Research Fund, Grant No. 196301051.
[1] W. Ehrenberg and R. E. Siday, Proc. Phys. Soc. London Sect. B 62, 8 (1949).

[2] Y. Aharonov and D. Bohm, Phys. Rev. 115, 485 (1959).

[3] For a review on synthetic gauge fields see: J. Dalibard, F. Gerbier, G. Juzeliūnas, and P. Öhberg, Rev. Mod. Phys. 83, 1523 (2011).

[4] Konstantinos Moulopoulos and Martha Constantinou, Phys. Rev. B 70, 235327 (2004)

[5] I.A. Shelykh, G. Pavlovic, D.D. Solnyshkov, and G. Malpuech, Phys. Rev. Lett. 102, 046407 (2009)

[6] D. R. Gulevich, D. V. Skryabin, A. P. Alodjants, and I. A. Shelykh, Phys. Rev. B 94, 115407 (2016).

[7] D. A. Zezyulin, D. R. Gulevich, D. V. Skryabin, and I. A. Shelykh, Phys. Rev. B 97, 161302(R) (2018)

[8] H. Deng, H. Haug, Y. Yamamoto, Rev. Mod. Phys. 82, 1489 (2010).
[9] D. Ballarini, D. Caputo, C. Sánchez Muñoz, M. De Giorgi, L. Dominici, M. H. Szymaǹska, K. West, L. N. Pfeiffer, G. Gigli, F. P. Laussy, and D. Sanvitto, Phys. Rev. Lett. 118, 215301 (2017).

[10] For a review on nonlinear and quantum collective properties of polariton systems see: Iacopo Carusotto and Cristiano Ciuti Rev. Mod. Phys. 85, 299 (2013)

[11] For a review on spin- related properties of polaritonic systems see: I.A. Shelykh, A.V. Kavokin, Yu.G. Rubo, T.C.H. Liew and G. Malpuech, Semicond. Sci. Technol. 25, 013001 (2010)

[12] K. Kusudo, N. Y. Kim, A. Löffler, S. Höfling, A. Forchel, and Y. Yamamoto, Phys. Rev. B 87, 214503 (2013).

[13] T. Jacqmin, I. Carusotto, I. Sagnes, M. Abbarchi, D. D. Solnyshkov, G. Malpuech, E. Galopin, A. Lemaître, J. Bloch, and A. Amo, Phys. Rev. Lett. 112, 116402 (2014). 
[14] M. Milićević, T. Ozawa, P. Andreakou, I. Carusotto, T. Jacqmin, E. Galopin, A. Lemaître, L. Le Gratiet, I. Sagnes, J. Bloch, and A Amo, 2D Mater. 2034012 (2015).

[15] A. V. Nalitov, D. D. Solnyshkov, and G. Malpuech, Phys. Rev. Lett. 114, 116401 (2015).

[16] D. R. Gulevich, D. Yudin, I. V. Iorsh, and I. A. Shelykh, Phys. Rev. B 94, 115437 (2016).

[17] O. Bleu, D.D. Solnyshkov, and G. Malpuech, Phys. Rev. B 95, 115415 (2017).

[18] O. Bleu, D.D. Solnyshkov, and G. Malpuech, Phys. Rev. B 95, 235431 (2017).

[19] C.E. Whittaker, E. Cancellieri, P.M. Walker, D.R. Gulevich, H. Schomerus, D. Vaitiekus, B. Royall, D.M. Whittaker, E. Clarke, I.V. Iorsh, I.A. Shelykh, M.S. Skolnick, and D.N. Krizhanovskii, Phys. Rev. Lett. 120, 097401 (2018)

[20] C. Ciuti, V. Savona, C. Piermarocchi, A. Quattropani, and P. Schwendimann, Phys. Rev. B 58, 7926 (1998)

[21] M. M. Glazov, H. Ouerdane, L. Pilozzi, G. Malpuech, A. V. Kavokin, and A. DAndrea, Phys. Rev. B 80, 155306 (2009)

[22] A. Amo, S. Pigeon, D. Sanvitto, V. G. Sala, R. Hivet, I. Carusotto, F. Pisanello, G. Leménager, R. Houdre,E. Giacobino, C. Ciuti, and A. Bramati, Science 332, 1167 (2011).

[23] M. Sich, D. N. Krizhanovskii, M. S. Skolnick, A. V. Gorbach, R. Hartley, D. V. Skryabin, E. A. Cerda-Méndez, K. Biermann, R. Hey, and P. V. Santos, Nature Photonics 6, 50 (2012).

[24] R. Hivet, H. Flayac, D. D. Solnyshkov, D. Tanese, T. Boulier, D. Andreoli, E. Giacobino, J. Bloch, A. Bramati, G. Malpuech and A. Amo, Nature Physics 8, 724 (2012)

[25] J. K. Chana, M. Sich, F. Fras, A. V. Gorbach, D. V. Skryabin, E. Cancellieri, E. A. Cerda-Méndez, K. Bier- mann, R. Hey, P. V. Santos, M. S. Skolnick, and D. N. Krizhanovskii, Physical Review Letters 115, 256401 (2015).

[26] D. R. Gulevich, D. Yudin, D. V. Skryabin, I. V. Iorsh, and I. A. Shelykh, Sci. Rep. 7, 1780 (2017).

[27] Yuri G. Rubo, Phys. Rev. Lett. 99, 106401 (2007)

[28] K. G. Lagoudakis, M. Wouters, M. Richard, A. Baas, I. Carusotto, R. Andre, L. S. Dang, and B. DeveaudPledran, Nature Physics 4, 706 (2008).

[29] G. Tosi, G. Christmann, N. G. Berloff, P. Tsotsis, T. Gao, Z. Hatzopoulos, P. G. Savvidis, and J. J. Baumberg, Nature Communications 3, 1243 (2012).

[30] T. Gao, O.A. Egorov, E. Estrecho, K. Winkler, M. Kamp, C. Schneider, S. Hofling, A.G. Truscott, and E.A. Ostrovskaya, Phys. Rev. Lett. 121, 225302 (2018)

[31] Min-Sik Kwon, Byoung Yong Oh, Su-Hyun Gong, JeHyung Kim, Hang Kyu Kang, Sooseok Kang, Jin Dong Song, Hyoungsoon Choi, and Yong-Hoon Cho, Phys. Rev. Lett. 122, 045302 (2019)

[32] Jonathan Keeling and Natalia G. Berloff, Phys. Rev. Lett. 100, 250401 (2008)

[33] Y. V. Kartashov, Chao Hang, G. Huang, and L. Torner, Optica 3, 1048 (2016).

[34] H. Sigurdsson, O. A. Egorov, X. Ma, I. A. Shelykh, and T. C. H. Liew, Phys. Rev. B 90, 014504 (2014)

[35] P. Lagoudakis and N. Berloff, New Journ. Phys. 19, 125008 (2017)

[36] T. C. H. Liew and Y. G. Rubo, Phys. Rev. B 97, 041302(R) (2018)

[37] A.V. Yulin, A.S. Desyatnikov, E.A. Ostrovskaya, Phys. Rev. B 94, 134310 (2016)

[38] H. Sakaguchi, B.A. Malomed, and D.V. Skryabin, New Journ. Phys. 19, 085003 (2017)

[39] A.V. Nalitov, H. Sigurdsson, S. Morina, Y.S. Krivosenko, I.V. Iorsh, Y.G. Rubo, A.V. Kavokin, and I.A. Shelykh, Phys. Rev. A 99, 033830 (2019) 